\title{
Magnetic Criticality Enhanced Hybrid Nanodiamond Thermometer under Ambient Conditions
}

\author{
Ning Wang, ${ }^{1}$ Gang-Qin Liu, ${ }^{1}$ Weng-Hang Leong, ${ }^{1}$ Hualing Zeng, ${ }^{3,1}$ Xi Feng, ${ }^{1}$ Si-Hong Li, ${ }^{1}$ Florian Dolde, ${ }^{4}$ \\ Helmut Fedder, ${ }^{4,6}$ Jörg Wrachtrup, ${ }^{4}$ Xiao-Dong Cui, ${ }^{5}$ Sen Yang, ${ }^{1,4}$ Quan Li, ${ }^{1,2,7, *}$ and Ren-Bao Liu ${ }^{1,2,7, \dagger}$ \\ ${ }^{1}$ Department of Physics, The Chinese University of Hong Kong, \\ Shatin, New Territories, Hong Kong, China \\ ${ }^{2}$ The Chinese University of Hong Kong Shenzhen Research Institute, Shenzhen 518100, China \\ ${ }^{3}$ ICQD, Hefei National Laboratory for Physical Science at the Microscale, \\ and CAS Key Laboratory of Strongly-coupled Quantum Matter Physics, \\ The University of Science and Technology of China, Hefei, Anhui 230026, China \\ ${ }^{4}$ Institute of Physics, Research Center SCoPE and IQST, University of Stuttgart, 70569 Stuttgart, Germany \\ ${ }^{5}$ Department of Physics, The University of Hong Kong, Hong Kong, China \\ ${ }^{6}$ Swabian Instruments GmbH, Frankenstr. 39, 71701 Schwieberdingen, Germany \\ ${ }^{7}$ Centre for Quantum Coherence, The Chinese University of Hong Kong, \\ Shatin, New Territories, Hong Kong, China
}

(Received 9 November 2017; revised manuscript received 9 January 2018; published 15 March 2018)

\begin{abstract}
Nitrogen-vacancy (NV) centers in diamond are attractive as quantum sensors owing to their superb coherence under ambient conditions. However, the NV center spin resonances are relatively insensitive to some important parameters such as temperature and pressure. Here we design and experimentally demonstrate a hybrid nanothermometer composed of NV centers and a magnetic nanoparticle (MNP), in which the temperature sensitivity is enhanced by the critical magnetization of the MNP near the ferromagnetic-paramagnetic transition temperature. The temperature susceptibility of the NV center spin resonance reaches $14 \mathrm{MHz} / \mathrm{K}$, nearly 200 times larger than that of bare NV centers. The sensitivity of a hybrid nanothermometer composed of a $\mathrm{Cu}_{1-x} \mathrm{Ni}_{x}$ MNP and a nanodiamond is measured to be $11 \mathrm{mK} / \sqrt{\mathrm{Hz}}$ under ambient conditions. The working range of the hybrid thermometer can be designed from cryogenic temperature to about $600 \mathrm{~K}$ by tuning the chemical composition of the $\mathrm{Cu}_{1-x} \mathrm{Ni}_{x} \mathrm{MNP}$. We demonstrate in situ detection of the magnetic phase transition of a single magnetic nanoparticle using the hybrid nanothermometer. This hybrid nanothermometer provides a novel approach to studying a broad range of thermal processes at nanoscales such as nanoplasmonics, heat-stimulated subcellular processes, and thermodynamics of nanosystems.
\end{abstract}

DOI: $10.1103 /$ PhysRevX.8.011042

Subject Areas: Magnetism, Nanophysics, Quantum Physics

\section{INTRODUCTION}

Nanoscale temperature sensing is important for studying a broad range of phenomena in physics, biology, and chemistry, such as the temperature heterogeneities in living cells [1-3], heat dissipation in nanocircuits [4], nanoplasmonics, and nanomagnetism (like thermal remanent magnetism of nanoparticles [5]). There have been a number of nanoscale temperature detection schemes [6], including scanning

\footnotetext{
*liquan@phy.cuhk.edu.hk

rbliu@cuhk.edu.hk
}

Published by the American Physical Society under the terms of the Creative Commons Attribution 4.0 International license. Further distribution of this work must maintain attribution to the author(s) and the published article's title, journal citation, and DOI. thermal microscopy [7,8], SQUID-based nanothermometer [9], and fluorescence thermometers [10] based on rare-earth nanoparticles [11], nanogels [12], dyes [13], or proteins [14]. However, these techniques are limited by various factors, such as low sensitivity (rare-earth nanoparticles), contactrelated artifacts (scanning thermal microscopy), fluorescence instability (dyes), or the requirement of extreme working conditions (SQUID-based nanothermometer). Nitrogenvacancy $(\mathrm{NV})$ centers in diamond is a promising nanosensor [15-24] owing to their atomic size and long spin coherence time $[25,26]$ under ambient conditions. The spin resonances of NV centers shift with temperature, providing a mechanism for atomic-scale temperature sensors [27]. However, the temperature dependence of $\mathrm{NV}$ center spin resonances, which results from the shift of the zero-field splitting $D$, is a small effect $(d D / d T \approx-74 \mathrm{kHz} / \mathrm{K})$ [27]. Therefore, even with single NV centers in ultrahigh purity bulk 
diamond sample and pulse control techniques, the sensitivity for temperature sensing is limited to a level of several $\mathrm{mK} / \sqrt{\mathrm{Hz}}($ Refs. [20-22]).

Here, we propose and demonstrate the strategy of hybridization [28-30] to improve the sensitivity of diamond nanothermometers by converting the temperature variation to a magnetic field change. NV centers have been demonstrated to be ultrasensitive to external magnetic field [15-17,31]. The magnetization of a magnetic nanoparticle (MNP) can be used to monitor its local temperature, and this mechanism becomes ultrasensitive when the temperature is close to the magnetic phase transition point (i.e., critical point) of the MNP. The sensitivity of a hybrid nanothermometer composed of a $\mathrm{Cu}_{1-x} \mathrm{Ni}_{x} \mathrm{MNP}$ and a nanodiamond under ambient conditions was measured to be $11 \mathrm{mK} / \sqrt{\mathrm{Hz}}$ (for comparison, the best sensitivity of nanodiamond thermometer achieved before was $130 \mathrm{mK} / \sqrt{\mathrm{Hz}}$; see Ref. [21]). The working range of the hybrid thermometer can be designed by choosing materials with different critical temperatures (e.g., via tuning the chemical composition of the $\left.\mathrm{Cu}_{1-x} \mathrm{Ni}_{x} \mathrm{MNP}\right)$. This hybrid

(a)

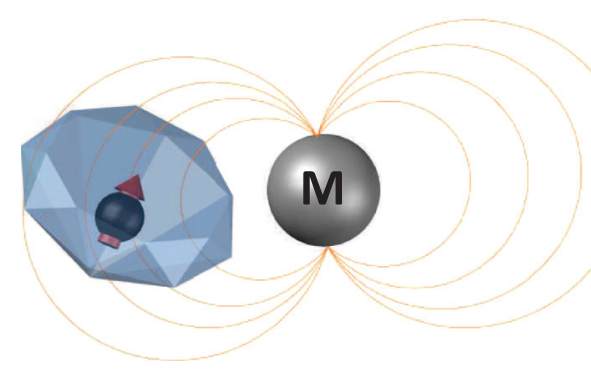

(b)

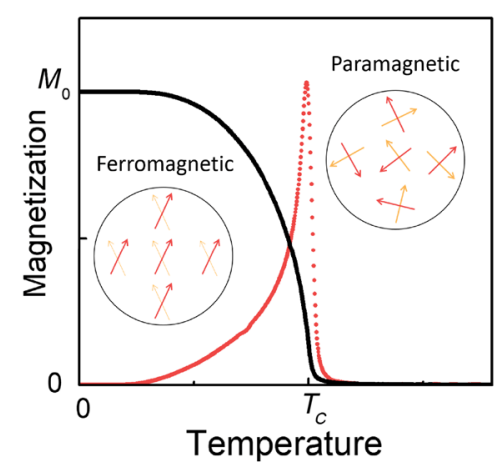

nanothermometer provides a novel approach to studying a broad range of thermal processes at nanoscales, such as nanoplasmonics, subcellular heat-stimulated processes, and thermodynamics of nanostructures.

\section{SCHEME OF THE HYBRID NANOSENSOR AND THEORETICAL SENSITIVITY}

The hybrid nanothermometer is composed of a fluorescent nanodiamond (FND) and a MNP, as shown in Fig. 1(a). The ground state of the NV center is a spin triplet $(S=1)$ with a zero-field splitting $D \approx 2.87 \mathrm{GHz}$ between the $m_{S}=0$ state and the $m_{S}= \pm 1$ states. The spin transitions of NV centers in the FND are shifted by the magnetic field from the MNP, $\mathbf{B}_{\mathrm{MNP}}(T)$. The Hamiltonian of a NV center can be written [32] as

$$
H_{\text {eff }}=D(T) S_{Z}^{2}+E\left(S_{X}^{2}-S_{Y}^{2}\right)+\gamma \mathbf{S} \cdot \mathbf{B}_{\mathrm{MNP}}(T),
$$

where $E$ is the lattice strain effect and $\gamma=28 \mathrm{MHz} / \mathrm{mT}$ is the electron gyromagnetic ratio. The field from the MNP $\mathbf{B}_{\mathrm{MNP}}(T)$ is determined by its magnetization $\mathbf{M}(T)$,

(c)

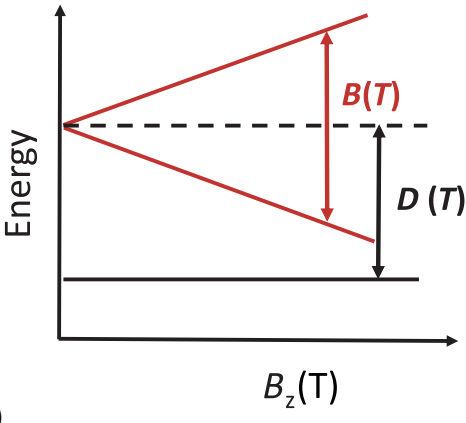

(d)

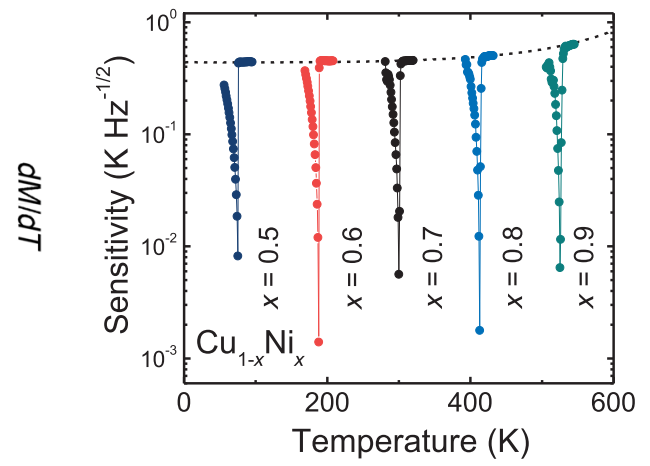

FIG. 1. Principle of magnetic criticality enhanced hybrid nanothermometers. (a) Schematic of a hybrid nanosensor, composed of a fluorescent nanodiamond (FND) and a magnetic nanoparticle (MNP). (b) The magnetic moment $M$ (black solid line) of the MNP decreases dramatically with temperature increasing to the ferromagnetic-paramagnetic transition, with the temperature susceptibility $d M / d T$ (red dotted line) peaking at the critical temperature $T_{C}$. The transition frequencies of the NV center spins in the FND depend sensitively on the magnetic moment of the MNP and hence the temperature. (c) Fine structure of the NV center ground state as a function of an axial magnetic field. The zero-field splitting $D$ varies with temperature with a rate $d D / d T \approx-74 \mathrm{kHz} / \mathrm{K}$. In the hybrid nanosensor, the magnetic field from the MNP $B_{\mathrm{MNP}}(T)$ results in a much more sensitive dependence of the transition frequencies on the temperature. (d) Theoretical sensitivity of the hybrid sensor composed of a FND and a $\mathrm{Cu}_{1-x} \mathrm{Ni}_{x} \mathrm{MNP}$ with different chemical composition $x$. The dashed line is the sensitivity of the bare NV-based thermal sensor. A spherical CuNi MNP with diameter of $200 \mathrm{~nm}$ is assumed. The FND is assumed of size $\approx 100 \mathrm{~nm}$ with $500 \mathrm{NV}$ centers (randomly distributed and orientated) and located $65 \mathrm{~nm}$ away from the MNP. 
which depends sensitively on the temperature when the temperature is close to the ferromagnetic-paramagnetic transition point [Fig. 1(b)]. Therefore, the spin resonances of the NV centers in the FND present abrupt dependence on temperature near the transition point of the MNP. The spin transition frequencies can be measured by optically detected magnetic resonance (ODMR). Previously, the temperature shift of zero-field splitting $d D / d T \approx-74 \mathrm{kHz} / \mathrm{K}$ was measured to extract the temperature change [20-22]. Here, the transition frequencies $\omega_{ \pm}$(for $m_{S}=0 \leftrightarrow m_{S}= \pm 1$ ) of the $\mathrm{NV}$ center spins are measured to extract the magnetization properties of the adjacent MNP [Fig. 1(c)], which serves as a transducer and amplifier of the local temperature variation.

The sensitivity of the hybrid sensor is determined by the magnetization of the MNP, the number of NV centers, the spin coherence, and the relative position and orientation between the two nanoparticles. FNDs with size $\approx 100 \mathrm{~nm}$ containing $500 \mathrm{NV}$ centers were used in experiments. As for MNPs, copper-nickel (CuNi) alloy nanoparticles were chosen for their chemical stability under ambient conditions. To estimate the sensitivity of the hybrid nanothermometer, we consider a spherical $\mathrm{Cu}_{0.30} \mathrm{Ni}_{0.70}$ MNP of diameter $200 \mathrm{~nm}$ located $65 \mathrm{~nm}$ away from a FND. The stability and repeatability of the magnetization can be ensured by the large anisotropic energy of the MNP with diameter of hundreds of nanometres. Taking into account the strain distribution ( $3 \mathrm{MHz}$ ), the random spatial and orientation distributions of the NV centers in the FND, and the magnetic field gradient from the MNP, the optimal sensitivity is estimated to be about $3 \mathrm{mK} / \sqrt{\mathrm{Hz}}$ (details in Note 1 of Supplemental Material [33], which includes Refs. [34-41]), as plotted by black dots in Fig. 1(d). This represents an improvement by 2 orders of magnitude from the sensitivity of a bare FND sensor (the previously record of similar FND sensors was $130 \mathrm{mK} / \sqrt{\mathrm{Hz}}$, as reported in Ref. [21]).

The optimal working range of the hybrid sensor, which is within $\approx 20^{\circ} \mathrm{C}$ below the Curie temperature, can be designed by choosing MNPs of different Curie temperatures. Taking $\mathrm{Cu}_{1-x} \mathrm{Ni}_{x}$ MNPs as an example, their Curie temperature is tunable from near 0 to $637 \mathrm{~K}$ with the nickel composition $x$ from $45 \%$ to $100 \%$ [42]. Together with the very broad working temperature of $\mathrm{NV}$ centers in diamond $[43,44]$, the hybrid sensor can be designed to fulfill a wide range of applications. Figure 1(d) presents the composition dependence of the sensitivity of the $\mathrm{Cu}_{1-x} \mathrm{Ni}_{x}$ MNP-based hybrid sensors in their optimal working ranges. The best temperature sensitivity for each hybrid sensor is better than $10 \mathrm{mK} / \sqrt{\mathrm{Hz}}$ from cryogenic temperature to $600 \mathrm{~K}$, which has taken into account the variation of the ODMR contrast with temperature [43].

\section{PROOF-OF-PRINCIPLE EXPERIMENT}

As a proof-of-principle demonstration of the magnetic criticality enhanced quantum sensing, we tested a hybrid sensor composed of a gadolinium (Gd) particle (with size $\approx 2 \mathrm{~mm}$ and mass $\approx 30 \mathrm{mg}$ ) and a single $\mathrm{NV}$ center in a bulk diamond $\left(3 \times 3 \times 1 \mathrm{~mm}^{3}\right)$. The distance between the $\mathrm{NV}$ center and the Gd particle is about $2 \mathrm{~mm}$ (comparable to the size of the Gd particle). Such a distance is chosen to simulate the configuration of a MNP coupled to a FND since the field from a magnetic particle, in the dipole approximation, is proportional to the total volume and inversely proportional to the cubic distance. Gd has the ferromagnetic-paramagnetic transition near room temperature $\left(T_{C} \approx 19^{\circ} \mathrm{C}\right)$ [45]. Continuous-wave (cw) ODMR measurement was carried out for temperature between $11^{\circ} \mathrm{C}$ and $37^{\circ} \mathrm{C}$ under a uniform external magnetic field $\left(B \approx 100 \mathrm{G}\right.$ with an angle of $20^{\circ}$ from the $\mathrm{NV}$ axis, estimated by the second-order frequency shift due to the transverse component of the magnetic field; for details of the method, see Note 3 of Supplemental Material [33]). The temperature was controlled by an incubator (INSTEC) and independently monitored by a Pt thermocouple.

Figure 2(a) presents the ODMR spectra of the hybrid sensor at various temperatures. The frequency shifts of the $m_{S}=0 \leftrightarrow m_{S}= \pm 1$ transitions [Fig. 2(b)] are enlarged near the critical temperature of $\mathrm{Gd}$, with $d \omega / d T$ reaching its maximum value $\approx 14 \mathrm{MHz} / \mathrm{K}$ at $19^{\circ} \mathrm{C}$. The induced magnetic moment is deduced from the ODMR spectrum, which is consistent with the magnetic moment of the same Gd particle measured by a vibrating sample magnetometer (Quantum Design; see Note 3 of Supplemental Material [33]). For comparison, the ODMR spectral shift of the same NV center with the Gd particle removed is shown in Fig. 2(c), which presents only a weak dependence on temperature, $d D / d T=-71 \pm 2 \mathrm{kHz} / \mathrm{K}$ [Fig. 2(d)], consistent with previous reports [27]. The magnetic criticality of the Gd particle induces an enhancement of spectral susceptibility to temperature by a factor of 200 . The magnetically enhanced temperature sensitivity of the NV center is evaluated with Eq. (A2) in the Appendix, with the experimentally measured factors including $d \omega / d T$, the spin resonance width, the contrast, and the photon counts. The experimentally realized temperature sensitivity of $45 \mathrm{mK} / \sqrt{\mathrm{Hz}}$ was limited by the weak photon counts ( $80 \mathrm{kps}$ per NV center) in our system, yet it is already 200 times better than that of a bare NV sensor under the same circumstance.

To achieve a better temperature sensitivity of the hybrid sensor, we replaced the single-NV bulk diamond with a FND containing ensemble NV centers (from Adamas Nanotechnologies, with about 500 NV centers per FND) to enhance the photon counts of the hybrid sensor. To show the dependence of the sensitivity on temperature, we measured the sensitivity at different temperatures with a photon count of $6 \mathrm{Mps}$. The resonant frequency $\omega_{-}$as a function of temperature is shown as an inset in Fig. 3(a) (with a sharp change near $T_{C}$ ). The sensitivity as a function of temperature, determined by Eq. (A2) in the Appendix, is shown in Fig. 3(a). 
(a)

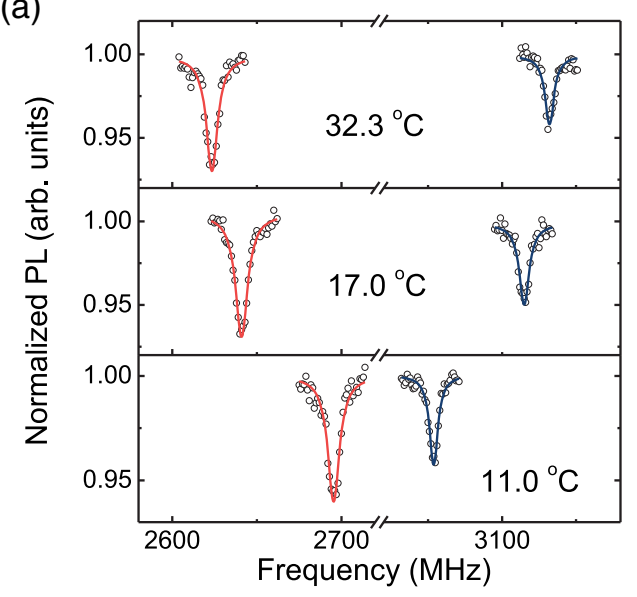

(b)

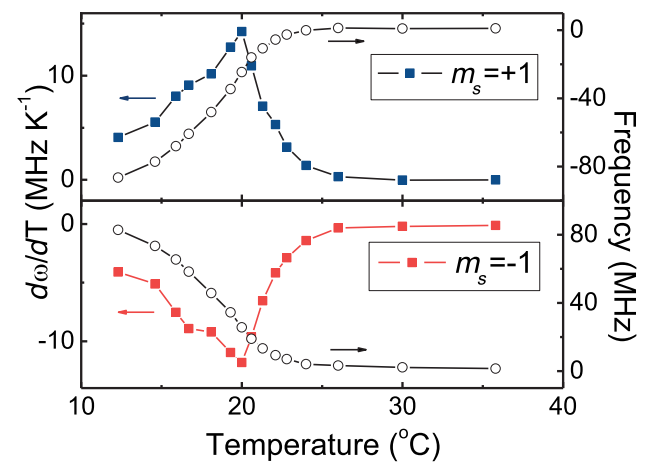

(c)

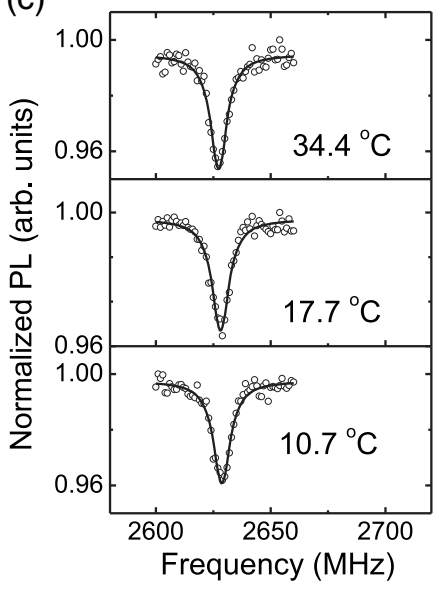

(d)

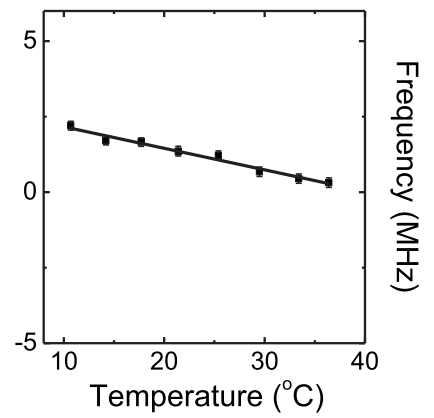

FIG. 2. Proof-of-principle demonstration of magnetic criticality enhanced temperature sensing. (a) The ODMR spectra of a NV center in a bulk diamond near a Gd particle at various temperatures near the critical point of $\mathrm{Gd}$. An external magnetic field of $100 \mathrm{G}$ was applied. With the criticality enhancement, the hybrid sensor shows a large spectral shift with temperature. Left (right) resonances [red (blue) lines] correspond to the $m_{S}=0 \leftrightarrow m_{S}=-1\left(m_{S}=+1\right)$ transitions. (b) The ODMR frequencies (black solid lines) of the hybrid sensor versus the temperature. The temperature susceptibility $d \omega_{ \pm} / d T$ (square dots) of the hybrid sensor reaches $\pm 14 \mathrm{MHz} / \mathrm{K}$ at the critical point $T_{C}=19^{\circ} \mathrm{C}$, which is 200 times larger than that of a bare NV sensor. (c) The ODMR spectra of the same NV center as in (a), but without the Gd particle attached. The bare NV center shows only a slight spectral shift of the $m_{S}=0 \leftrightarrow m_{S}=-1$ transition under the same temperature change as in (a). In (a) and (c), the dots are experimental data and the lines are the Lorentzian fitting. (d) The ODMR frequency of the bare NV center as a function of the temperature, which has a slope of $-71 \pm 2 \mathrm{kHz} / \mathrm{K}$.

(a)

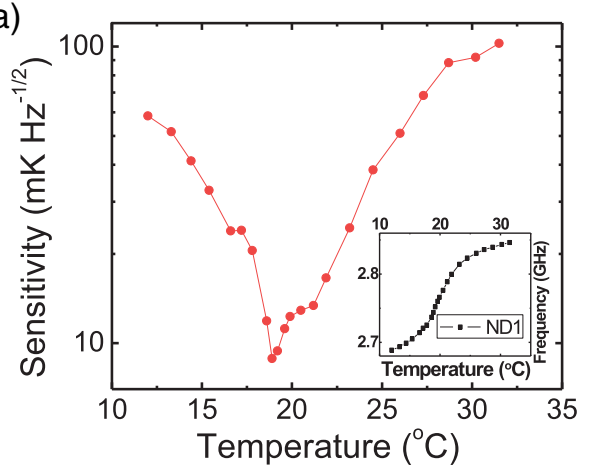

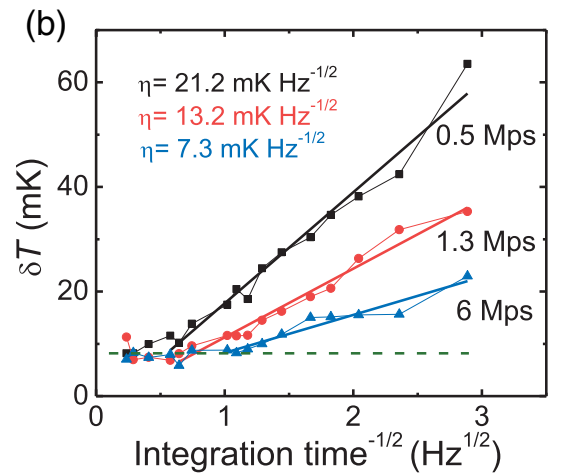

FIG. 3. Sensitivity of a magnetic criticality enhanced Gd-FND temperature sensor. (a) The sensitivity of the hybrid sensor at various temperatures. The red dots are sensitivity determined by Eq. (A2) using the experimentally measured photon counts, ODMR contrast, $d \omega / d T$, and spin resonance width $\Delta \omega$. The laser power is chosen such that the photon count is $\approx 6$ Mps. The optimal sensitivity $\approx 7.3 \mathrm{mK} / \sqrt{\mathrm{Hz}}$ is achieved at $T_{C}=19^{\circ} \mathrm{C}$. Inset: Frequency shift of the Gd-FND sensor as a function of temperature under an external magnetic field of $100 \mathrm{G}$. (b) The standard deviation of temperature measurement as a function of data acquisition time for various photon counts (due to different laser powers) using the three-point method. The slopes of the curves give the shot-noise-limited sensitivity of the hybrid sensor. The green dashed line corresponds to the temperature stability of the incubator. 
For optical detection sensing, a fundamental limitation to sensitivity is the shot noise of photon counts [32]. To check whether the sensitivity in our system is limited by photon shot noise, we measured the temperature with various data acquisition time. The three-point cw ODMR method is adapted from Refs. [20,46] for real-time temperature determination (see Appendix A and Note 2 of Supplemental Material [33] for details). The temperature range was chosen near the optimal value $\left(T_{C}\right.$ of $\left.\mathrm{Gd}\right)$. Figure 3(b) shows the standard deviation $\delta T$ of the temperature measurement as a function of the data acquisition time. With long enough integration time for all three photon count rates (corresponding to three different excitation laser powers), the minimum measurable temperature variation $\delta T$ approaches a lower bound of about $10 \mathrm{mK}$, which was limited by the temperature stability of the incubator. The linear dependence of $\delta T$ on the inverse square root of integration time gives the photon shot-noise-limited sensitivity. As expected, higher photon counts lead to higher sensitivity. A shot-noise-limited sensitivity of $7.3 \mathrm{mK} / \sqrt{\mathrm{Hz}}$ was achieved with the configuration of $\mathrm{Gd}$ particle and FND.

\section{NANOTHERMOMETER-CUNi MNP AND FND}

Next, we demonstrate that the criticality enhanced sensing scheme works at nanoscale. Since gadolinium nanoparticles are easily oxidized under ambient conditions, we use copper-nickel (CuNi) MNPs instead of $\mathrm{Gd}$ nanoparticles in the hybrid nanosensor. As shown in Fig. 4(a), the pattern matching between the confocal image and the transmission electron microscopy (TEM) image identifies a CuNi MNP located in the proximity of a FND. The temperature change of the CuNi MNP is induced by in situ laser heating of the carbon film on the TEM grid (the direct heating of the MNP and the FND by laser and microwave is negligible; see Note 4 of Supplemental Material [33]). Since the heating laser spot ( $300 \mathrm{~nm}$ in diameter) is larger than the distance between the two particles (less than $100 \mathrm{~nm}$ ), we assume that the temperature at the FND and (a)

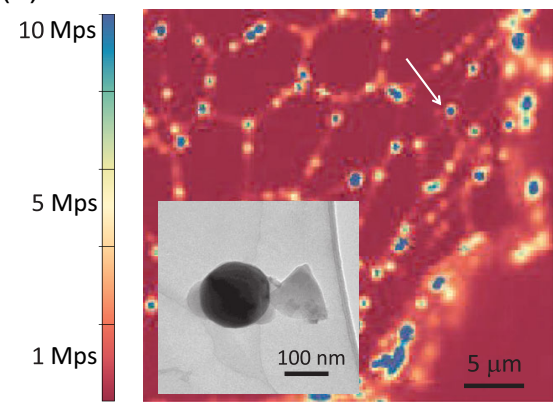

(c)

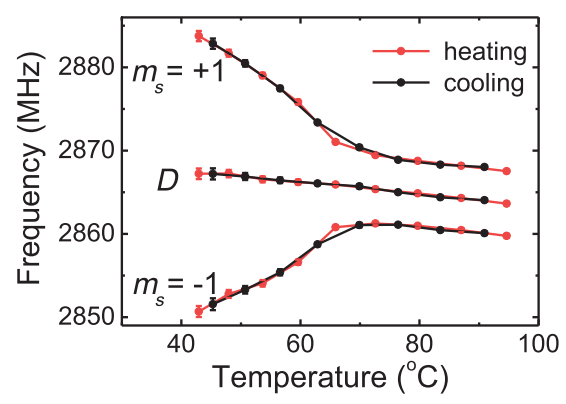

(b)

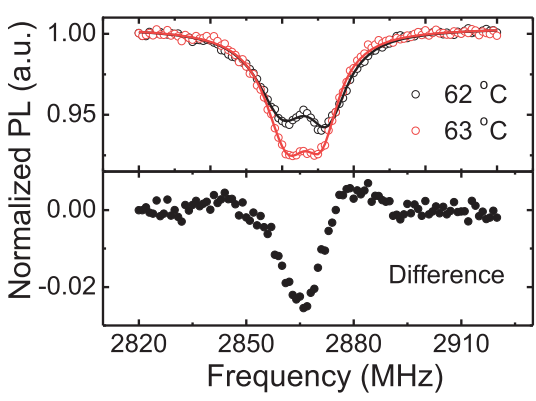

(d)

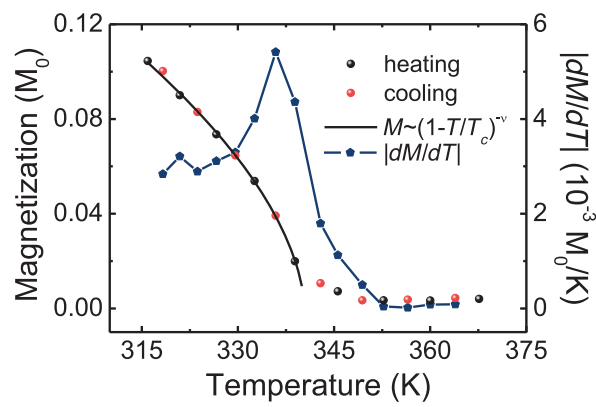

FIG. 4. Magnetic criticality enhanced hybrid nanothermometer. The nanothermometer is composed of a FND (with about 500 NV centers inside) and a nearby $\mathrm{Cu}_{1-x} \mathrm{Ni}_{x}$ MNP. (a) Confocal image of the hybrid sensor (at the arrowhead). The inset shows the TEM image of the measured sensor, in which the CuNi MNP is black and the FND is transparent. (b) Typical ODMR spectra of the hybrid sensor at two different temperatures. The splitting is caused by the magnetic field from the MNP since no external magnetic field was applied. Lower panel is the signal difference of the two ODMR spectra in the upper panel. (c) ODMR resonant frequencies of the $m_{S}=0 \leftrightarrow m_{S}= \pm 1$ transitions as functions of temperature. The zero-field splitting $D$ is obtained by averaging the two frequencies. Both heating (red dots) and cooling (black dots) processes were measured. (d) The spontaneous magnetization of the MNP extracted from the ODMR spectrum. The red and black dots are measured in the heating and cooling processes, respectively. The blue curve shows the temperature susceptibility of the magnetization. The transition temperature of this nanoparticle is about $340 \mathrm{~K}$, which is consistent with its chemical composition $\left(\mathrm{Cu}_{0.26} \mathrm{Ni}_{0.74}\right)$ measured by energy-dispersive X-ray spectroscopy in the TEM. 
the MNP is identical. The temperature was calibrated by the zero-field splitting $D$ (details in Appendix $C$ and Note 4 of Supplemental Material [33]) of FND. During the nanothermometer experiments, no external magnetic field was applied.

The temperature response of the nanothermometer is determined by measuring the resonant transition frequencies $\omega_{ \pm}$of NV centers. The gradient of the magnetic field from the CuNi MNP, the spatial distribution of NV centers in the FND, and the four possible crystallographic orientations of NV centers in diamond bring extra broadening in the ODMR spectra, as shown in Fig. 4(b), upper panel. Nevertheless, the ODMR dips due to Zeeman splitting are well discernible. For all different laser powers (hence, temperatures), the ODMR spectra are well consistent with the theoretical simulation that includes the Zeeman splitting, the MNP gradient-induced broadening, and the strain distribution of ensemble NV centers in the FND (see Note 4 of Supplemental Material [33]).

The frequency shift induced by the magnetization of the MNP is reversible when the temperature is scanned back and forth around the critical temperature of the $\mathrm{CuNi}$ MNP, as shown in Fig. 4(c). The reversibility is ascribed to the large anisotropy energy of $\mathrm{CuNi}$ nanoparticles. Furthermore, we found this hybrid sensor is very stable. It shows negligible changes in the long-term measurement lasting for near one month (see Note 4 of Supplemental Material [33] for more information). From the resonance frequencies, the magnetization of the CuNi MNP at different temperatures can be determined by numerical simulation, which agrees well with the critical behavior of $\mathrm{CuNi}$ [Fig. 4(d)]. The Curie temperature of this CuNi MNP is determined to be about $340 \mathrm{~K}$, consistent with its composition $\left(\mathrm{Cu}_{0.26} \mathrm{Ni}_{0.74}\right)$ measured by the energy-dispersive $\mathrm{X}$-ray spectroscopy in TEM [42]. The consistency verifies that the temperatures of the MNP and the FND are identical to each other under $\mathrm{cw}$ laser heating.

\section{NANOTHERMOMETER SENSITIVITY MEASUREMENT}

In the ODMR spectra of the nanosensor, due to the magnetic field gradient and NV center distribution in the FND, the changes in line shape (both the line width and contrast) are much more significant than the frequency shift with temperature, especially for temperatures close to the critical point. As illustrated in Fig. 4(b), upper panel, with $1{ }^{\circ} \mathrm{C}$ temperature variation near $T_{C}\left(67^{\circ} \mathrm{C}\right)$, the ODMR line shape changes significantly. From the difference between the two spectra, we find that the most sensitive point for temperature sensing (maximum contrast change $d S / d T$ ) is close to the middle point of the two dips [Fig. 4(b), lower panel]. According to Eq. (A1) in the Appendix, a sensitivity of $11 \mathrm{mK} / \sqrt{\mathrm{Hz}}$ was realized at this point $(d S / d T=0.025 / \mathrm{K}$ at $\omega=2866 \mathrm{MHz}$ with photon counts $L=12 \mathrm{Mps}$ ). This temperature sensitivity is close to the theoretical optimal value $(3 \mathrm{mK} / \sqrt{\mathrm{Hz}})$ of a FND-CuNi-MNP hybrid sensor shown in Fig. 1(d). Note that in this configuration, the magnetic gradient-induced broadening is greater than the difference of the Zeeman splittings between NV centers along different crystallographic directions. Therefore, the sensitivity of the hybrid sensor is relatively independent of the magnetization axis of the MNP (see Note 1 of Supplemental Material [33] for more discussions).

With the high temperature sensitivity and a three-point method (see the Appendix for details), we can monitor realtime temperature variation with high time resolution. With the three-point method, the minimum measurable temperature variations for different integration time are presented in Fig. 5(a), which gives the shot-noise-limited sensitivity of the hybrid sensor $(20 \mathrm{mK} / \sqrt{\mathrm{Hz}})$. The light gray region is limited by the temperature stability of the experimental system. Furthermore, we use in situ laser heating to generate a fast temperature variation at the sample position by modulating the intensity of the excitation laser (the (a)

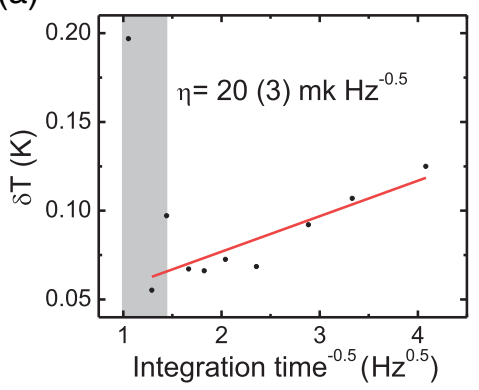

(b)

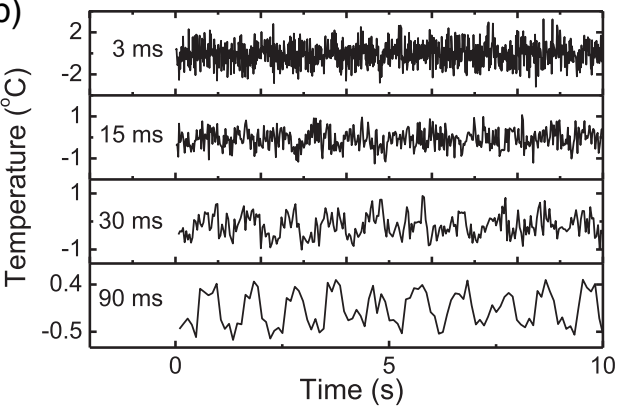

FIG. 5. Shot-noise-limited sensitivity and real-time temperature tracking at nanoscale. The hybrid sensor [the one in Fig. 4(a)] was used to monitor fast temperature variations at nanoscale. The local temperature was controlled by in situ laser heating, which has tunable amplitude. (a) Dependence of the temperature standard deviation on data acquisition time with the laser power (and hence temperature) kept constant. The shot-noise-limited sensitivity is derived from the slope of the curve. (b) With the laser power being modulated, the temperature variation (with frequency of $1 \mathrm{~Hz}$ and amplitude of $0.45^{\circ} \mathrm{C}$ ) was tracked with various acquisition time $(3,15,30$, and $90 \mathrm{~ms}$ per data point, from top to bottom). The $0.9{ }^{\circ} \mathrm{C}$ temperature variation is distinguishable with an integration time as small as $15 \mathrm{~ms}$ per data point. 
temperature is calibrated by the $D$ shift as a function of the laser power above Curie temperature). As shown in Fig. 5(b), with different integration time, the periodic temperature $(1 \mathrm{~Hz})$ alternation between two values $\left(63 \pm 0.45^{\circ} \mathrm{C}\right)$ is tracked. With an integration time $3 \mathrm{~ms}$ (1 $\mathrm{ms}$ for measurement at each frequency point), the $0.9^{\circ} \mathrm{C}$ temperature variation is faintly visible, which approaches the best shot-noise-limited sensitivity. When increasing the integration time, the shot noise of photon counts is suppressed and the signal-to-noise ratio of the temperature signal becomes better. With an integration time of $90 \mathrm{~ms}$, the $0.9^{\circ} \mathrm{C}$ temperature vibration is well discerned in real-time sensing. Note that the measured temperature signal resembles the shape of the driving laser pulse, indicating good reversibility and stability of this hybrid sensor.

\section{DISCUSSION}

The temperature sensitivity can be further improved by using a NV center of longer spin coherence time and employing pulse control protocols. Figure 6(a) shows the configuration of a hybrid sensor composed of a $200 \mathrm{~nm}$ $\mathrm{Cu}_{1-x} \mathrm{Ni}_{x} \mathrm{MNP}$ on a diamond nanopillar with a single NV center under zero magnetic field. The diamond nanopillar has the advantages of longer spin coherence time $T_{2}^{*}$ (similar to single NV centers in bulk diamond, which is longer than $1 \mu \mathrm{s}$ in diamond of natural isotope abundance $[24,32]$ ), high photon collection efficiency (with photon counts $\approx 1$ Mps [47]), and absence of extra broadening due to the magnetic field gradient of the MNP. To be specific, we assume the magnetic field from the MNP is along the
NV axis. The sensitivity as a function of temperature is plotted as red dots in Fig. 6(c), and the required microwave pulse sequence for the Ramsey interference between the $\left|m_{S}=+1\right\rangle$ and $\left|m_{S}=-1\right\rangle$ states is shown in Fig. 6(b). A sensitivity of $4 \mu \mathrm{K} / \sqrt{\mathrm{Hz}}$ can be achieved near the Curie temperature of the CuNi MNP. In comparison with the simulation results of the bare NV thermometer [with echo control protocol adopted [20-22], as shown by blue triangles in Fig. 6(c)], the sensitivity can be improved by $2-3$ orders of magnitude with our criticality enhanced scheme. For magnetic fields away from the NV axis, the sensitivity is only modestly reduced from the optimal orientation. In the case that the magnetic field from the MNP is perpendicular to the $\mathrm{NV}$ axis (the worst scenario), the first-order Zeeman shift becomes zero, and the resonance splitting will have a second-order dependence $\left(\sim \gamma^{2} B^{2} / D\right)$. The sensitivity due to the second-order effect is reduced by a factor of $\gamma B / D$, but for the MNP and the single NV center in nanopillar under consideration, a sensitivity of $80 \mu \mathrm{K} / \sqrt{\mathrm{Hz}}$ is still achievable (see Fig. S1F in Note 1 of Supplemental Material [33] for numerical simulations).

With its superb sensitivity and designable working temperature range, the hybrid nanothermometer enables in situ and real-time observation of nonuniform heat dissipation of microelectronic or nanoelectronic devices [4], nanoplasmonics, subcellular thermal processes, and chemical reaction at nanoscale [48]. The FND-CuNi MNP nanothermometer can work under zero magnetic field. That allows the setup to be miniaturized and the sensor to be applied to liquid environments where the rotation of the sensor under an external field would cause complications (a)

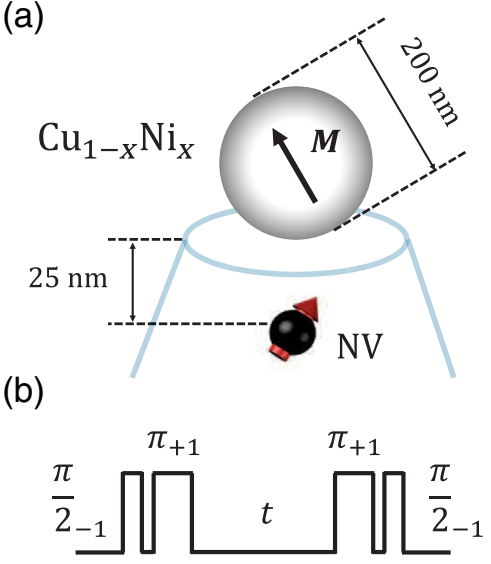

(c)

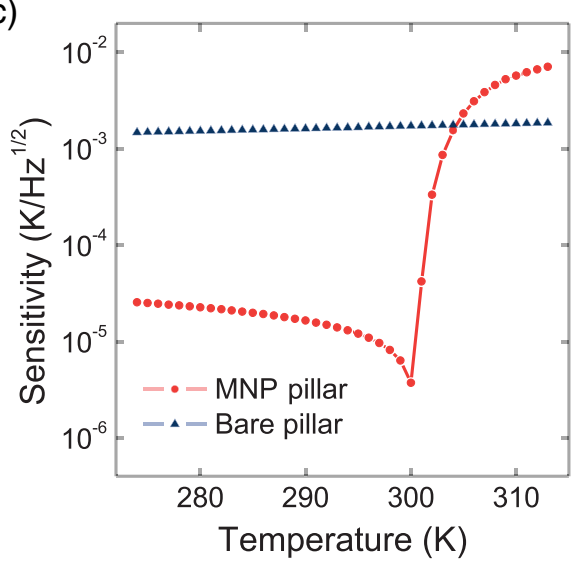

FIG. 6. Theoretical sensitivity of a hybrid nanothermometer composed of a MNP and a single NV center in a diamond nanopillar. The nanostructure-enhanced fluorescence collection efficiency and long coherence times of single NV centers in diamond nanopillars greatly improve the temperature sensitivity. (a) Schematic of the hybrid sensor that contains a MNP on the top of a diamond nanopillar with a single NV center $25 \mathrm{~nm}$ below the surface. (b) Microwave control pulse sequence for Ramsey interference between the $m_{S}=-1$ and $m_{S}=+1$ states. The $\pi / 2$ pulses and $\pi$ pulses are applied on the $m_{S}=0 \leftrightarrow m_{S}=-1$ and $m_{S}=0 \leftrightarrow m_{S}=+1$ transitions, respectively. (c) Sensitivity versus temperature. The red circles are for the hybrid sensor and the blue triangles are for a bare NV center sensor under the pulse control scheme in Refs. [20-22]. The NV spin coherence times are assumed as $T_{2}^{*} \approx 1.9 \mu \mathrm{s}$ (used for the hybrid sensor) and $T_{2} \approx 85 \mu \mathrm{s}$ [22] (used for the bare NV center sensor), and the photon count rate is $L \approx 1.7 \mathrm{Mps}$ [47]. 
due to variation of the ODMR spectra. Furthermore, nanodiamond-based sensors are biocompatible and robust against systematic errors such as fluorescence fluctuation in complicated intracellular environments. These unique merits enable direct monitoring of cell metabolism, and may help to clarify the controversies on temperature imaging in single living cells [1-3].

\section{ACKNOWLEDGMENTS}

This work was supported by the National Basic Research Program of China (973 Program) under Grant No. 2014CB921402, Hong Kong Research Grants Council-Collaborative Research Fund CUHK4/CRF/12G, Hong Kong RGC/GRF 14319016, and The Chinese University of Hong Kong Vice Chancellor's One-off Discretionary Fund. H. Z. acknowledges the support from China Government Youth 1000-Plan Talent Program, the Fundamental Research Funds for the Central Universities under No. WK2340000082, and National Natural Science Foundation of China under Grant No. 11674295.

R.-B.L. and Q. L. conceived the idea, designed the project, and supervised the research, W.-H. L. carried out the theoretical studies and numerical simulation, N. W., G.-Q. L., H.Z., Q. L., and R.-B. L. designed the systems and measurements, G.-Q. L., N. W., H. Z., S.-H. L., F. D., S. Y., X.-D. C., and J. W. set up the ODMR system, N. W. and G.-Q. L. carried out the experiments, N. W. and X. F. processed the samples, F.X. characterized MNPs and FNDs on TEM grids, N. W., G.-Q. L., H.Z., W.-H. L., X. F., Q. L., and R.-B. L. analyzed the data, N. W. wrote the paper with input from W.-H.L., G.-Q.L., and H.Z., and all authors commented on the manuscript. N. W., G.-Q. L., W.-H.L., and H.Z. contributed equally to this work.

\section{APPENDIX}

\section{Sensitivity - Theoretical estimation and experimental measurements}

Magnetization of the $\mathrm{Cu}_{0.3} \mathrm{Ni}_{0.7}$ alloy MNP is calculated with a mean-field theory. Transition frequencies of the NV center spins are obtained by diagonalizing the effective Hamiltonian in Eq. (1). For ensemble NV centers in a FND, cw ODMR is considered for temperature sensing. The sensitivity is [15]

$$
\eta_{\mathrm{cw}}=\frac{1}{\sqrt{L}}\left|\frac{d S(\omega)}{d T}\right|_{\max }^{-1},
$$

where $S(\omega)$ is the normalized ODMR spectrum and $L$ is the photon count rate. For details, see Note 1 of Supplemental Material [33]. If the ODMR spectrum is of Lorentzian shape and only resonant frequencies change with temperature, the sensitivity can be optimized by choosing the frequency near the half-height of the resonance. The result is

$$
\eta_{\mathrm{cw}}=\frac{4}{3 \sqrt{3}} \frac{\Delta \omega}{C \sqrt{L}|d \omega / d T|},
$$

where $\Delta \omega$ is the line width and $C$ is the ODMR contrast. Three-point ODMR measurements were employed for the demonstration of shot-noise-limited sensitivity. One point was chosen as the reference microwave frequency to normalize the laser fluctuation for long-term measurement. The sensitivity evaluated from the three-point method is larger by $\sqrt{3 / 2}$ than the sensitivities evaluated from Eqs. (A1) and (A2), since $1 / 3$ of the acquisition time was used to take reference data (details in Note 2 of Supplemental Material [33]). The standard deviation of temperature $\delta T$ as a function of the square root of the data acquisition time $\Delta t$ determines the temperature sensitivity, $\eta=\delta T \sqrt{\Delta t}$. For the three-point method, we apply different evaluation methods to extract the temperature information for different shapes of the ODMR spectra. In the Gd-FND experiment, $F=\left(N_{1}-N_{2}\right) /\left(N_{1}+N_{2}-2 N_{3}\right)$ was measured to extract the temperature information $\left(N_{1,2}\right.$ is the photon counts near the frequencies of full width at half maximum in corresponding acquisition times, and $N_{3}$ is the photon count for the reference frequency). In the $\mathrm{CuNi}$ FND nanosensor measurement, $F=\left(N_{1}+N_{2}-2 N_{3}\right) / N_{3}$ was measured $\left(N_{1,2}\right.$ is the photon counts for the frequencies where the contrast changes the most, and $N_{3}$ is photon counts for reference). Details are in Note 2 of Supplemental Material [33].

\section{Sample preparation}

The bulk diamond (from Element Six) is a high-purity type-IIa sample with a natural $1.1 \%{ }^{13} \mathrm{C}$ abundance. The FNDs (from Adamas Nanotechnologies) are of type Ib, prepared by high-pressure high-temperature synthesis. Each FND contains about 500 NV centers as claimed by Adamas and confirmed by the high fluorescence rate. The $\mathrm{CuNi}$ MNPs were prepared by ball milling of micron-size $\mathrm{Cu}_{0.30} \mathrm{Ni}_{0.70}$ alloy (from Sigma-Aldrich) or of micron-size $\mathrm{Cu}$ and $\mathrm{Ni}$ particles with a designed ratio. The composition of the MNPs (hence, the Curie temperature) can be readily tuned by choosing the ratio of $\mathrm{Ni}$ and $\mathrm{Cu}$ particles. The FNDs and the CuNi MNPs were dispersed in ethanol separately. Then the two solutions were subsequently dropped on a TEM gold grid. The size, composition, and relative positions of the two types of nanoparticles were characterized by TEM techniques. The featured patterns of carbon film on the TEM grid were used to match the confocal and TEM images.

\section{Experimental setup}

We used a home-built confocal microscope for imaging and ODMR measurement. NV center spins are optically 
pumped by a $532 \mathrm{~nm}$ laser, manipulated by resonant microwave fields applied through a $25 \mu \mathrm{m}$ diameter gold wire, and detected via spin-state-dependent fluorescence measurements. The power of the microwave is adjusted to maximize the ODMR contrast but without extra heating effect and is kept unchanged through the experiments. For FNDs, the width of ODMR spectra is mainly determined by the inhomogeneous broadening of ensemble NV centers (which is much greater than the power broadening induced by the laser excitation). For the CuNi-FND hybrid sensor, the nanoscale magnetic field gradient causes extra broadening to the ODMR spectra (see Notes 1 and 4 of Supplemental Material [33] for details). An incubator from INSTEC was used in the Gd-related experiments to control the temperature with the stability of sub $10 \mathrm{mK}$. A Pt thermocouple was placed near the Gd particle to monitor the local temperature. The heating and cooling rate of the incubator is $0.5^{\circ} \mathrm{C} / \mathrm{min}$, and ODMR measurements were carried out after the sample reached its thermal equilibrium state (in about $20 \mathrm{~min}$ ). In the experiments on the CuNi MNP-FND sensor, the MNPs were in situ heated by light absorption of the carbon film on the TEM grid. The local temperature of the hybrid sensor was tuned by laser intensity, which was controlled by the acoustic optical modulator before the fiber entrance of the microscope. The liquid environment of the hybrid sensor (oil immersed) stabilizes the laser heating effect. The measured thermal stability of laser heating on the TEM grid is better than $0.5^{\circ} \mathrm{C}$, in the temperature range from room temperature $\left(25^{\circ} \mathrm{C}\right)$ to about $100^{\circ} \mathrm{C}$. The local temperature of the hybrid sensor has linear dependence on the laser power. And it is calibrated above the $T_{C}$ of the CuNi MNP in the absence of an external field, at which the MNP is demagnetized and the zero-field splitting $D$ becomes a good parameter to deduce the local temperature (since the effect of transverse magnetic field is absent). Under cw excitation of different laser power, two-peak Lorentzian fitting is used to extract the resonance frequencies $\left(\omega_{+}\right.$and $\left.\omega_{-}\right)$of the ODMR spectra, as shown in Fig. 4(c), and $D=\left(\omega_{+}+\omega_{-}\right) / 2$ is used to deduce the local temperature shift of the hybrid sensor (data points above $T_{C}$ ). The temperature calibration is further confirmed by numerical simulation; see Note 4 of Supplemental Material [33] for details.

[1] G. Baffou, H. Rigneault, D. Marguet, and L. Jullien, A Critique of Methods for Temperature Imaging in Single Cells, Nat. Methods 11, 899 (2014).

[2] S. Kiyonaka, R. Sakaguchi, I. Hamachi, T. Morii, T. Yoshizaki, and Y. Mori, Validating Subcellular Thermal Changes Revealed by Fluorescent Thermosensors, Nat. Methods 12, 801 (2015).

[3] M. Suzuki, V. Zeeb, S. Arai, K. Oyama, and S. Ishiwata, The $10^{5}$ Gap Issue between Calculation and Measurement in Single-Cell Thermometry, Nat. Methods 12, 802 (2015).
[4] M. Mecklenburg, W. A. Hubbard, E. R. White, R. Dhall, S. B. Cronin, S. Aloni, and B. C. Regan, Nanoscale Temperature Mapping in Operating Microelectronic Devices, Science 347, 629 (2015).

[5] D. J. Dunlop, Magnetic Recording in Rocks, Phys. Today 65, No. 6, 31 (2012).

[6] Y. Yue and X. Wang, Nanoscale Thermal Probing, Nano Rev. 3, 11586 (2012).

[7] A. Majumdar, Scanning Thermal Microscopy, Annu. Rev. Mater. Sci. 29, 505 (1999).

[8] F. Menges, P. Mensch, H. Schmid, H. Riel, A. Stemmer, and B. Gotsmann, Temperature Mapping of Operating Nanoscale Devices by Scanning Probe Thermometry, Nat. Commun. 7, 10874 (2016).

[9] D. Halbertal, J. Cuppens, M. B. Shalom, L. Embon, N. Shadmi, Y. Anahory, H. R. Naren, J. Sarkar, A. Uri, Y. Ronen, Y. Myasoedov, L. S. Levitov, E. Joselevich, A. K. Geim, and E. Zeldov, Nanoscale Thermal Imaging of Dissipation in Quantum Systems, Nature (London) 539, 407 (2016).

[10] D. Jaque and F. Vetrone, Luminescence Nanothermometry, Nanoscale 4, 4301 (2012).

[11] E. C. Ximendes, W. Q. Santos, U. Rocha, U. K. Kagola, F. Sanz-Rodríguez, N. Fernández, A. D. S. Gouveia-Neto, D. Bravo, A. M. Domingo, B. del Rosal, C. D. S. Brites, L. D. Carlos, D. Jaque, and C. Jacinto, Unveiling In Vivo Subcutaneous Thermal Dynamics by Infrared Luminescent Nanothermometers, Nano Lett. 16, 1695 (2016).

[12] C. Gota, K. Okabe, T. Funatsu, Y. Harada, and S. Uchiyama, Hydrophilic Fluorescent Nanogel Thermometer for Intracellular Thermometry, J. Am. Chem. Soc. 131, 2766 (2009).

[13] K. Okabe, N. Inada, C. Gota, Y. Harada, T. Funatsu, and S. Uchiyama, Intracellular Temperature Mapping with a Fluorescent Polymeric Thermometer and Fluorescence Lifetime Imaging Microscopy, Nat. Commun. 3, 705 (2012).

[14] J. S. Donner, S. A. Thompson, M. P. Kreuzer, G. Baffou, and R. Quidant, Mapping Intracellular Temperature Using Green Fluorescent Protein, Nano Lett. 12, 2107 (2012).

[15] J. M. Taylor, P. Cappellaro, L. Childress, L. Jiang, D. Budker, P. R. Hemmer, A. Yacoby, R. Walsworth, and M. D. Lukin, High-Sensitivity Diamond Magnetometer with Nanoscale Resolution, Nat. Phys. 4, 810 (2008).

[16] J. R. Maze, P. L. Stanwix, J. S. Hodges, S. Hong, J. M. Taylor, P. Cappellaro, L. Jiang, M. V. G. Dutt, E. Togan, A. S. Zibrov, A. Yacoby, R. L. Walsworth, and M. D. Lukin, Nanoscale Magnetic Sensing with an Individual Electronic Spin in Diamond, Nature (London) 455, 644 (2008).

[17] G. Balasubramanian, I. Y. Chan, R. Kolesov, M. Al-Hmoud, J. Tisler, C. Shin, C. Kim, A. Wojcik, P. R. Hemmer, A. Krueger, T. Hanke, A. Leitenstorfer, R. Bratschitsch, F. Jelezko, and J. Wrachtrup, Nanoscale Imaging Magnetometry with Diamond Spins under Ambient Conditions, Nature (London) 455, 648 (2008).

[18] F. Dolde, H. Fedder, M. W. Doherty, T. Nöbauer, F. Rempp, G. Balasubramanian, T. Wolf, F. Reinhard, L. C. L. Hollenberg, F. Jelezko, and J. Wrachtrup, Electric-Field Sensing Using Single Diamond Spins, Nat. Phys. 7, 459 (2011).

[19] M. W. Doherty, V. V. Struzhkin, D. A. Simpson, L. P. McGuinness, Y. Meng, A. Stacey, T. J. Karle, R. J. Hemley, 
N. B. Manson, L. C. L. Hollenberg, and S. Prawer, Electronic Properties and Metrology Applications of the Diamond $\mathrm{NV}^{-}$Center under Pressure, Phys. Rev. Lett. 112, 047601 (2014).

[20] G. Kucsko, P. C. Maurer, N. Y. Yao, M. Kubo, H. J. Noh, P. K. Lo, H. Park, and M.D. Lukin, Nanometre-Scale Thermometry in a Living Cell, Nature (London) 500, 54 (2013).

[21] P. Neumann, I. Jakobi, F. Dolde, C. Burk, R. Reuter, G. Waldherr, J. Honert, T. Wolf, A. Brunner, J. H. Shim, D. Suter, H. Sumiya, J. Isoya, and J. Wrachtrup, HighPrecision Nanoscale Temperature Sensing Using Single Defects in Diamond, Nano Lett. 13, 2738 (2013).

[22] D. M. Toyli, C. F. de Las Casas, D. J. Christle, V. V. Dobrovitski, and D. D. Awschalom, Fluorescence Thermometry Enhanced by the Quantum Coherence of Single Spins in Diamond, Proc. Natl. Acad. Sci. U.S.A. 110, 8417 (2013).

[23] T. Plakhotnik, M. W. Doherty, J. H. Cole, R. Chapman, and N. B. Manson, All-Optical Thermometry and Thermal Properties of the Optically Detected Spin Resonances of the NV Center in Nanodiamond, Nano Lett. 14, 4989 (2014).

[24] R. Schirhagl, K. Chang, M. Loretz, and C. L. Degen, Nitrogen-Vacancy Centers in Diamond: Nanoscale Sensors for Physics and Biology, Annu. Rev. Phys. Chem. 65, 83 (2014).

[25] G. Balasubramanian, P. Neumann, D. Twitchen, M. Markham, R. Kolesov, N. Mizuochi, J. Isoya, J. Achard, J. Beck, J. Tissler, V. Jacques, P. R. Hemmer, F. Jelezko, and J. Wrachtrup, Ultralong Spin Coherence Time in Isotopically Engineered Diamond, Nat. Mater. 8, 383 (2009).

[26] N. Bar-Gill, L. Pham, A. Jarmola, D. Budker, and R. Walsworth, Solid-State Electronic Spin Coherence Time Approaching One Second, Nat. Commun. 4, 1743 (2013).

[27] V. M. Acosta, E. Bauch, M. P. Ledbetter, A. Waxman, L.-S. Bouchard, and D. Budker, Temperature Dependence of the Nitrogen-Vacancy Magnetic Resonance in Diamond, Phys. Rev. Lett. 104, 070801 (2010).

[28] M. Schaffry, E. M. Gauger, J. J. L. Morton, and S. C. Benjamin, Proposed Spin Amplification for Magnetic Sensors Employing Crystal Defects, Phys. Rev. Lett. 107, 207210 (2011).

[29] J. Cai, F. Jelezko, and M. B. Plenio, Hybrid Sensors Based on Colour Centres in Diamond and Piezoactive Layers, Nat. Commun. 5, 4065 (2014).

[30] L. Trifunovic, F. L. Pedrocchi, S. Hoffman, P. Maletinsky, A. Yacoby, and D. Loss, High-Efficiency Resonant Amplification of Weak Magnetic Fields for Single Spin Magnetometry at Room Temperature, Nat. Nanotechnol. 10, 541 (2015).

[31] T. Wolf, P. Neumann, K. Nakamura, H. Sumiya, T. Ohshima, J. Isoya, and J. Wrachtrup, Subpicotesla Diamond Magnetometry, Phys. Rev. X 5, 041001 (2015).

[32] L. Rondin, J.-P. Tetienne, T. Hingant, J.-F. Roch, P. Maletinsky, and V. Jacques, Magnetometry with Nitrogen-Vacancy Defects in Diamond, Rep. Prog. Phys. 77, 056503 (2014).

[33] See Supplemental Material at http://link.aps.org/ supplemental/10.1103/PhysRevX.8.011042 for details of the system, the experimental and theoretical methods, and the supplemental data of the hybrid sensors, which includes Refs. [34-41].

[34] A. P. Guimarães, Principles of Nanomagnetism (Springer, Berlin, 2009).

[35] W. F. Brown, Jr, Thermal Fluctuations of a Single-Domain Particle, Phys. Rev. 130, 1677 (1963).

[36] R. S. Schoenfeld and W. Harneit, Real Time Magnetic Field Sensing and Imaging Using a Single Spin in Diamond, Phys. Rev. Lett. 106, 030802 (2011).

[37] R. J. Epstein, F. M. Mendoza, Y. K. Kato, and D. D. Awschalom, Anisotropic Interactions of a Single Spin and Dark-Spin Spectroscopy in Diamond, Nat. Phys. 1, 94 (2005).

[38] B. A. Myers, A. Das, M. C. Dartiailh, K. Ohno, D. D. Awschalom, and A. C. Bleszynski Jayich, Probing Surface Noise with Depth-Calibrated Spins in Diamond, Phys. Rev. Lett. 113, 027602 (2014).

[39] Y. Romach, C. Müller, T. Unden, L. J. Rogers, T. Isoda, K. M. Itoh, M. Markham, A. Stacey, J. Meijer, S. Pezzagna, B. Naydenov, L. P. McGuinness, N. Bar-Gill, and F. Jelezko, Spectroscopy of Surface-Induced Noise Using Shallow Spins in Diamond, Phys. Rev. Lett. 114, 017601 (2015).

[40] J. Wang, W. Zhang, J. Zhang, J. You, Y. Li, G. Guo, F. Feng, X. Song, L. Lou, W. Zhu, and G. Wang, Coherence Times of Precise Depth Controlled NV Centers in Diamond, Nanoscale 8, 5780 (2016).

[41] M. W. Doherty, J. Michl, F. Dolde, I. Jakobi, P. Neumann, N. B. Manson, and J. Wrachtrup, Measuring the Defect Structure Orientation of a Single $\mathrm{NV}^{-}$Centre in Diamond, New J. Phys. 16, 063067 (2014).

[42] S. A. Ahern, M. J. C. Martin, and W. Sucksmith, The Spontaneous Magnetization of Nickel + Copper Alloys, Proc. R. Soc. A 248, 145 (1958).

[43] D. M. Toyli, D. J. Christle, A. Alkauskas, B. B. Buckley, C. G. Van de Walle, and D. D. Awschalom, Measurement and Control of Single Nitrogen-Vacancy Center Spins above 600 K, Phys. Rev. X 2, 031001 (2012).

[44] A. Jarmola, V. M. Acosta, K. Jensen, S. Chemerisov, and D. Budker, Temperature- and Magnetic-Field-Dependent Longitudinal Spin Relaxation in Nitrogen-Vacancy Ensembles in Diamond, Phys. Rev. Lett. 108, 197601 (2012).

[45] S. Y. Dan'kov, A. M. Tishin, V. K. Pecharsky, and K. A. Gschneidner, Magnetic Phase Transitions and the Magnetothermal Properties of Gadolinium, Phys. Rev. B 57, 3478 (1998).

[46] Y.-K. Tzeng, P.-C. Tsai, H.-Y. Liu, O. Y. Chen, H. Hsu, F.-G. Yee, M.-S. Chang, and H.-C. Chang, Time-Resolved Luminescence Nanothermometry with Nitrogen-Vacancy Centers in Nanodiamonds, Nano Lett. 15, 3945 (2015).

[47] S. A. Momenzadeh, R. J. Stöhr, F. F. de Oliveira, A. Brunner, A. Denisenko, S. Yang, F. Reinhard, and J. Wrachtrup, Nanoengineered Diamond Waveguide as a Robust Bright Platform for Nanomagnetometry Using Shallow Nitrogen Vacancy Centers, Nano Lett. 15, 165 (2015).

[48] C. Y. Jin, Z. Li, R. S. Williams, K.-C. Lee, and I. Park, Localized Temperature and Chemical Reaction Control in Nanoscale Space by Nanowire Array, Nano Lett. 11, 4818 (2011). 